\title{
Ultimate Robust Performance Control of Rigid Robot Manipulators using Interval Arithmetic
}

\author{
Andrea Giusti and Matthias Althoff
}

\begin{abstract}
We propose a new method for controlling rigid robot manipulators guaranteeing that a user-defined tracking performance is ultimately met while facing uncertain system dynamics and input disturbance. Our control approach is based on the passivity-based control technique and eliminates the limitations of existing methods (e.g. impractical tuning to avoid chattering and difficult estimation of uncertainty bounds of disturbances) through the use of interval arithmetic. Our controller guarantees to meet the tracking performance when considering input disturbance with unknown bounds. Additionally, our approach can be used for on-the-fly controller synthesis, which makes it especially appealing for robust control of modular and reconfigurable robot manipulators. Finally, we present simulation results and a comparison with the $r-\alpha$ tracking controller that shows the superior performance of our proposed method when considering a realistic scenario with limited sampling rate.
\end{abstract}

\section{INTRODUCTION}

Model-based control methods represent a key technology to enhance the motion-control performance of robot manipulators [1]. Obviously, the required models only match the real system dynamics with varying degree. Additionally, possible changes of end-effectors and payloads influence the overall system dynamics. New control laws or several tuning iterations may thus be required for guaranteeing high motion-control performance. In order to overcome these shortcomings, the robust control of robot manipulators has attracted researchers for decades. A survey that describes works until the early ' 90 s can be found in [2] and more recent contributions are [3]-[9], among others. Nowadays, effective classical and more recent approaches are well documented in textbooks such as [10]-[13].

Known robust control methods for robot manipulators that guarantee tracking, where the error asymptotically converges to zero, rely on discontinuous control laws that are often difficult to implement in practice [14], [15]. In fact, the resulting control laws lead to an undesired chattering behaviour of the torque command. A recent contribution that solves a long-standing inconsistency in the design of the robust controllers for classical approaches is in [9], where a more reasonable and effective design methodology is proposed. The resulting controller, however, is still discontinuous. Smoothed versions of such controllers can be implemented to guarantee continuity of the control law as presented in [15]. The consequence is that the asymptotic convergence to zero of the tracking error is lost. As an alternative,

The authors are with the Department of Informatics, Technical University of Munich (TUM), 85748 Garching, Germany. Corresponding email: \{giusti, althoff\}@in.tum.de the authors of [15] develop a theory for practical stability of uncertain systems introducing the concept of uniform ultimate boundedness of the trajectories that has been widely adopted in successive literature. One of the most effective methods that incorporates this smoothing idea is [3] that does not suffer from the inconsistencies highlighted by [9] and its performance has been experimentally evaluated in [16]. A remarkable approach for robust control that is not based on the smoothing of a discontinuous control law is in [5], where authors present the $r$ - $\alpha$ tracking controller, which is a simple controller that allows tracking trajectories with a user-defined rate of convergence $\alpha$ of the tracking error to a specified tolerance $r$.

Although the research on robust control for rigid robot manipulators is relatively mature, we identify limitations that may be crucial for the practical implementation of existing methods. In practice, these approaches may suffer from a difficult estimation of bounds of disturbances due to imperfect knowledge of the system dynamics. These bounds typically depend on the desired trajectory and the state of the manipulator. This aspect is also highlighted in [3], where a controller that exploits the property of linear parametrization of the dynamical model [11, Sec. 7.2] is proposed to address this limitation. However, that controller is based on the idea of smoothing a discontinuous control law as proposed in [15]. A limitation appears when a specific tracking performance is required. In fact, as also suggested in [10], a scalar tuning parameter for smoothing the control law should be selected large enough to avoid the chattering phenomena which is typical of discontinuous control laws, but enlarging this parameter also enlarges the bounds that enclose the trajectories, making the tuning process difficult. An approach that takes inspiration from the above mentioned method is presented in [12, Sec. 2.4.2]. In that case, the controller does not approach a discontinuous control law when increasing the required tracking performance. Both discussed approaches in [3] and [12, Sec. 2.4.2] do not directly consider an external disturbance and, in contrast to our work, are not suitable for ultimate robust performance control with unknown bounds of the input disturbance. Additionally, since they exploit the property of the linear parametrization of the dynamical model, they require to obtain and compute the regressor matrix, which is not a requirement of our method.

We propose a novel approach for robust control of rigid robot manipulators which guarantees that a user-defined tracking performance is ultimately met even though the dynamical parameters of the system are uncertain and input disturbances with unknown bounded norm are considered. 
Our method is based on passivity-based control and uses interval arithmetic to overcome the difficulties for estimating the bounds in other approaches with the additional advantage that our design can be easily automated. This latter feature is particularly valuable for on-the-fly controller synthesis, which is especially useful for modular robots whose configuration frequently changes [17]. In contrast to our proposed approach, the robust controllers proposed so far which require the estimation of uncertainty bounds of the disturbances, have no formal guarantees of their properties since this estimation is not over-approximative.

The rest of this paper is structured as follows. In Sec. II we describe the control problem for ultimate robust performance control in detail. Our proposed approach is presented in Sec. III. Simulation results are shown and discussed in Sec. IV, followed by the conclusion in Sec. V.

\section{PROBLEM DESCRIPTION}

We consider a rigid robot manipulator composed of $N$ serially connected links with uncertain dynamical parameters. For brevity of notation we omit time dependance of time-varying variables hereafter, except when we want to stress time dependance particularly. Let $\mathbf{q} \in \mathbb{R}^{N}$ be the vector of generalized coordinates, $\mathbf{u} \in \mathbb{R}^{N}$ the vector of the joint forces/torques, $\mathbf{d} \in \mathbb{R}^{N}$ the input disturbance vector and

$$
\Delta=\left(m_{1}, \ldots, m_{N}, c_{x, 1}, \ldots, c_{z, N}, I_{x x, 1}, \ldots, I_{z z, N}\right)^{T},
$$

the vector of the parameters of the links where the mass, the coordinates of the center of mass and the inertia tensor of the $i^{t h}$ link are respectively denoted by

$$
m_{i}, \mathbf{c}_{i}=\left(\begin{array}{c}
c_{x, i} \\
c_{y, i} \\
c_{z, i}
\end{array}\right) \text { and } \mathbf{I}_{i}=\left(\begin{array}{ccc}
I_{x x, i} & -I_{x y, i} & -I_{x z, i} \\
* & I_{y y, i} & -I_{y z, i} \\
* & * & I_{z z, i}
\end{array}\right) .
$$

The mathematical model that describes the dynamics of the considered system can be written as follows (see [11, Ch. 7]):

$$
\mathbf{M}(\mathbf{q}, \Delta) \ddot{\mathbf{q}}+\mathbf{C}(\mathbf{q}, \dot{\mathbf{q}}, \Delta) \dot{\mathbf{q}}+\mathbf{g}(\mathbf{q}, \Delta)=\mathbf{u}+\mathbf{d},
$$

where $\mathbf{M}(\mathbf{q}, \Delta) \in \mathbb{R}^{N \times N}$ is the symmetric and positive definite inertia matrix, $\mathbf{C}(\mathbf{q}, \dot{\mathbf{q}}, \Delta) \dot{\mathbf{q}} \in \mathbb{R}^{N}$ is the vector of Coriolis and centrifugal terms, and $\mathbf{g}(\mathbf{q}, \Delta) \in \mathbb{R}^{N}$ the vector of gravity terms. It is important to recall that, with a suitable factorization of $\mathbf{C}(\mathbf{q}, \dot{\mathbf{q}}, \Delta) \in \mathbb{R}^{N \times N}$, the matrix $\mathbf{N}(\mathbf{q}, \dot{\mathbf{q}}, \Delta)=$ $\dot{\mathbf{M}}(\mathbf{q}, \Delta)-2 \mathbf{C}(\mathbf{q}, \dot{\mathbf{q}}, \Delta)$ is skew-symmetric, and therefore:

$$
\mathbf{x}^{T} \mathbf{N}(\mathbf{q}, \dot{\mathbf{q}}, \Delta) \mathbf{x}=0, \quad \forall \mathbf{x} \in \mathbb{R}^{N} .
$$

We assume that: a) the model structure is known, b) a nominal vector of dynamical parameters $\Delta_{0}$ is available, $c$ ) the amount of the uncertainty of each component of $\Delta$ is known, and $d$ ) the input disturbance has bounded norm $\beta_{d}$ (we consider both cases of known and unknown bound $\beta_{d}$ ). All norms in this work are Euclidean norms. Throughout this paper we do not consider friction for simplicity and without loss of generality as it becomes evident later. A central definition follows, that allows us to specify in detail the control problem we face.
Definition 1 (Ultimate robust performance): Let $\mathbf{q}_{d} \in \mathbb{R}^{N}$ be the desired trajectory in joint space, $\mathbf{q} \in \mathbb{R}^{N}$ the vector of the generalized coordinates and $\varepsilon>0$ a desired upper bound of the trajectory tracking error norm. A controlled robot manipulator with uncertain dynamics has ultimate robust performance if there exists a finite time $t_{1} \geq 0$ such that:

$$
\left\|\mathbf{q}_{d}-\mathbf{q}\right\|<\varepsilon, \forall t \geq t_{1} .
$$

When $\varepsilon$ is unspecified we say that the solutions are uniformly ultimately bounded with the same meaning and terminology of [15]. Within the previous assumptions, we face the problem of designing a continuous control law for the system of (1) that provides ultimate robust performance for a userdefined $\varepsilon>0$.

\section{PROPOSED METHOD}

As previously introduced, our proposed control approach is based on results from passivity-based control. Therefore, the control command is computed using the nominal model information and an additional term $v \in \mathbb{R}^{N}$ that enhances robustness (see e.g. [13]):

$$
\mathbf{u}=\mathbf{M}\left(\mathbf{q}, \Delta_{0}\right) \ddot{\mathbf{q}}_{a}+\mathbf{C}\left(\mathbf{q}, \dot{\mathbf{q}}, \Delta_{0}\right) \dot{\mathbf{q}}_{a}+\mathbf{g}\left(\mathbf{q}, \Delta_{0}\right)-v,
$$

where $\Delta_{0}$ denotes the vector of the nominal dynamical parameters and

$$
\begin{array}{cc}
\dot{\mathbf{q}}_{a}=\dot{\mathbf{q}}_{d}+\mathbf{K}_{r} \tilde{\mathbf{q}}, & \tilde{\mathbf{q}}=\mathbf{q}_{d}-\mathbf{q}, \\
\ddot{\mathbf{q}}_{a}=\ddot{\mathbf{q}}_{d}+\mathbf{K}_{r} \dot{\tilde{\mathbf{q}}}, & \dot{\tilde{\mathbf{q}}}=\dot{\mathbf{q}}_{d}-\dot{\mathbf{q}},
\end{array}
$$

with $\mathbf{K}_{r}$ being a diagonal positive definite matrix of proper dimension. Applying the control law of (4) to the system of (1), results after rearrangement in (see [12, Sec. 2.4.2]):

$$
\mathbf{M}(\mathbf{q}, \Delta) \dot{\mathbf{r}}+\mathbf{C}(\mathbf{q}, \dot{\mathbf{q}}, \Delta) \mathbf{r}=v+\mathbf{w}\left(\mathbf{q}, \dot{\mathbf{q}}, \dot{\mathbf{q}}_{a}, \ddot{\mathbf{q}}_{a}, \mathbf{d}, \Delta\right),
$$

where

$$
\mathbf{r}=\dot{\tilde{\mathbf{q}}}+\mathbf{K}_{r} \tilde{\mathbf{q}}
$$

and $\mathbf{w}\left(\mathbf{q}, \dot{\mathbf{q}}, \dot{\mathbf{q}}_{a}, \ddot{\mathbf{q}}_{a}, \mathbf{d}, \Delta\right)$ is a disturbance vector due the external disturbance inputs and due to the imperfect knowledge of the system dynamics. This term can be expressed as

$$
\begin{aligned}
\mathbf{w}\left(\mathbf{q}, \dot{\mathbf{q}}, \dot{\mathbf{q}}_{a}, \ddot{\mathbf{q}}_{a}, \mathbf{d}, \Delta\right)=\underset{\mathbf{M}}{(\mathbf{q}, \Delta)} \ddot{\mathbf{q}}_{a}+ \\
\tilde{\mathbf{C}}(\mathbf{q}, \dot{\mathbf{q}}, \Delta) \dot{\mathbf{q}}_{a}+\tilde{\mathbf{g}}(\mathbf{q}, \Delta)-\mathbf{d},
\end{aligned}
$$

where

$$
\begin{gathered}
\tilde{\mathbf{M}}(\mathbf{q}, \Delta)=\mathbf{M}(\mathbf{q}, \Delta)-\mathbf{M}_{0}(\mathbf{q}), \mathbf{M}_{0}(\mathbf{q})=\mathbf{M}\left(\mathbf{q}, \Delta_{0}\right), \\
\tilde{\mathbf{C}}(\mathbf{q}, \dot{\mathbf{q}}, \Delta)=\mathbf{C}(\mathbf{q}, \dot{\mathbf{q}}, \Delta)-\mathbf{C}_{0}(\mathbf{q}, \dot{\mathbf{q}}), \\
\mathbf{C}_{0}(\mathbf{q}, \dot{\mathbf{q}})=\mathbf{C}\left(\mathbf{q}, \dot{\mathbf{q}}, \Delta_{0}\right), \\
\tilde{\mathbf{g}}(\mathbf{q}, \Delta)=\mathbf{g}(\mathbf{q}, \Delta)-\mathbf{g}_{0}(\mathbf{q}), \mathbf{g}_{0}(\mathbf{q})=\mathbf{g}\left(\mathbf{q}, \Delta_{0}\right) .
\end{gathered}
$$

For now we assume a known bound on the input disturbance that will be relaxed later. Since we also know the amount of the uncertainty of the properties of each link, we can bound them by multidimensional intervals that are defined as follows.

Definition 2 (Multidimensional interval): A multidimensional interval is a set of real numbers defined as

$[\mathbf{x}]:=[\underline{\mathbf{x}}, \overline{\mathbf{x}}], \quad \underline{\mathbf{x}} \in \mathbb{R}^{n}, \overline{\mathbf{x}} \in \mathbb{R}^{n}, \underline{x}_{i} \leq \bar{x}_{i}$, for all $i=1, \ldots, n$. 
We denote the scalar case by $x$ instead of $\mathbf{x}$, and we use $\underline{x}$ and $\bar{x}$ to denote the infimum and supremum of an interval $[x]$, respectively.

It is now possible to define the interval of uncertain values of the mass, the inertia tensor and the coordinates of the center of mass of the $i^{t h}$ link as $\left[m_{i}\right],\left[\mathbf{I}_{i}\right]$ and $\left[\mathbf{c}_{i}\right]$, respectively. It is worth noting that uncertain payload parameters can be included in those of the last link. The interval vector of the uncertain parameters can be written as:

$$
[\Delta]=\left(\left[m_{1}\right] \ldots,\left[m_{N}\right],\left[c_{x, 1}\right], \ldots,\left[c_{z, N}\right],\left[I_{x x, 1}\right], \ldots,\left[I_{z z, N}\right]\right)^{T} .
$$

Since our method is based on the on-line evaluation of an interval-valued function, we provide the following definition.

Definition 3 (Interval-valued function): Given $\mathbf{z}: \mathbb{R}^{n} \rightarrow$ $\mathbb{R}^{m}$, its interval evaluation over a set $[\mathbf{x}]$ is defined as:

$$
\mathbf{z}([\mathbf{x}]):=\{\mathbf{z}(\mathbf{x}) \mid \mathbf{x} \in[\mathbf{x}]\} .
$$

We are now ready to state that for $\mathbf{d} \in[\mathbf{d}]$ and $\Delta \in[\Delta]$ :

$$
\mathbf{w}\left(\mathbf{q}, \dot{\mathbf{q}}, \dot{\mathbf{q}}_{a}, \ddot{\mathbf{q}}_{a}, \mathbf{d}, \Delta\right) \subseteq[\Phi]=\mathbf{w}\left(\mathbf{q}, \dot{\mathbf{q}}, \dot{\mathbf{q}}_{a}, \ddot{\mathbf{q}}_{a},[\mathbf{d}],[\Delta]\right),
$$

where the inclusion relation of (8) can be inferred straightforwardly form Def. 3 . We measure the size of $[\Phi]$ using the following definition.

Definition 4 (Measure of the worst-case disturbance):

Let $\mathbb{I} \mathbb{R}^{N}$ be the set of all real interval vectors and $\rho: \mathbb{R}^{N} \rightarrow \mathbb{R}^{N}$. We define the measure of the worst-case disturbance $[\Phi]$ as:

$$
\rho([\Phi])=\max (|\underline{\Phi}|,|\bar{\Phi}|)
$$

where the $\max (\cdot)$ operator is applied element-wise. For later derivations, we require continuity of $\rho([\Phi])$, which is shown in the following proposition.

Proposition 1: Let $\mathbf{q}, \dot{\mathbf{q}}, \dot{\mathbf{q}}_{a}, \ddot{\mathbf{q}}_{a}$ be continuous over time, then for all $[\mathbf{d}] \in \mathbb{I R}^{N}$ and $[\Delta] \in \mathbb{I} \mathbb{R}^{M}$ (where $M$ is the dimension of $\Delta$ ), the measure of the worst-case disturbance $\rho([\Phi])$ is continuous over time.

Proof: Let us recall that $[\Phi]=\mathbf{w}\left(\mathbf{q}, \dot{\mathbf{q}}, \dot{\mathbf{q}}_{a}, \ddot{\mathbf{q}}_{a},[\mathbf{d}],[\Delta]\right)$ (see (8)). For a given $\mathbf{q}, \dot{\mathbf{q}}, \dot{\mathbf{q}}_{a}, \ddot{\mathbf{q}}_{a}$, we can choose $\mathbf{d}^{*} \in[\mathbf{d}]$ and $\Delta^{*} \in[\Delta]$ such that $\bar{\Phi}$ is maximal (or $\underline{\Phi}$ is minimal). Since $\mathbf{q}$, $\dot{\mathbf{q}}, \dot{\mathbf{q}}_{a}, \ddot{\mathbf{q}}_{a}$ are assumed to be continuous, $\mathbf{w}\left(\mathbf{q}, \dot{\mathbf{q}}, \dot{\mathbf{q}}_{a}, \ddot{\mathbf{q}}_{a}, \mathbf{d}^{*}, \Delta^{*}\right)$ is continuous guaranteeing in turn continuity of $|\bar{\Phi}|$ (or $|\underline{\Phi}|$ ). Since the max operator between two continuous functions preserves continuity, $\rho([\Phi])$ is continuous.

Lemma 1: For all q, $\dot{\mathbf{q}}, \dot{\mathbf{q}}_{a}, \ddot{\mathbf{q}}_{a} \in \mathbb{R}^{N}, \mathbf{d} \in[\mathbf{d}]$ and $\Delta \in[\Delta]$

$$
\rho_{i}([\Phi]) \geq w_{i}\left(\mathbf{q}, \dot{\mathbf{q}}, \dot{\mathbf{q}}_{a}, \ddot{\mathbf{q}}_{a}, \mathbf{d}, \Delta\right),
$$

where the subscript $i$ denotes the $i^{\text {th }}$ component of the respective vectors.

Proof: We prove by contradiction. Let us suppose that $\rho_{i}([\Phi])<w_{i}\left(\mathbf{q}, \dot{\mathbf{q}}, \dot{\mathbf{q}}_{a}, \ddot{\mathbf{q}}_{a}, \mathbf{d}, \Delta\right)$. Then we have that $\max \left(\left|\underline{\Phi_{i}}\right|,\left|\overline{\Phi_{i}}\right|\right)<w_{i}\left(\mathbf{q}, \dot{\mathbf{q}}, \dot{\mathbf{q}}_{a}, \ddot{\mathbf{q}}_{a}, \mathbf{d}, \Delta\right)$ which shows the contradiction since by definition of $\left[\Phi_{i}\right]$, we have that $\underline{\Phi}_{i} \leq$ $w_{i}\left(\mathbf{q}, \dot{\mathbf{q}}, \dot{\mathbf{q}}_{a}, \ddot{\mathbf{q}}_{a}, \mathbf{d}, \Delta\right) \leq \bar{\Phi}_{i}$, for all $\mathbf{d} \in[\mathbf{d}]$ and $\Delta \in[\Delta]$.

The following theorem introduces the use of $\rho([\Phi])$ for feedback control and allows us to avoid the difficult estimation of the uncertainty bounds as required in previous work mentioned in Sec. I.
Theorem 1: Given two positive increasing functions $\kappa(t)$ and $\varphi(t)$ with $\kappa_{P} \geq 1$ and $\varphi_{P} \geq 1$ as their respective minimum, the trajectories $\mathbf{r}$ in (5), are uniformly ultimately bounded when using the feedback control law

$$
v=-(\kappa(t)\|\rho([\Phi])\|+\varphi(t)) \mathbf{r} .
$$

(5) as:

Proof: Let us consider the Lyapunov candidate for

$$
V(\mathbf{r})=\frac{1}{2} \mathbf{r}^{T} \mathbf{M}(\mathbf{q}, \Delta) \mathbf{r}
$$

which is positive for all $\mathbf{r} \neq \mathbf{0}$ since the inertia matrix is positive definite for all $\mathbf{q}$. The derivative of this function can be written as

$$
\begin{aligned}
& \dot{V}(\mathbf{r})= \mathbf{r}^{T} \mathbf{M}(\mathbf{q}, \Delta) \dot{\mathbf{r}}+\frac{1}{2} \mathbf{r}^{T} \dot{\mathbf{M}}(\mathbf{q}, \Delta) \mathbf{r} \\
& \stackrel{(5)}{=} \mathbf{r}^{T}\left(v+\mathbf{w}\left(\mathbf{q}, \dot{\mathbf{q}}, \dot{\mathbf{q}}_{a}, \ddot{\mathbf{q}}_{a}, \mathbf{d}, \Delta\right)\right) \\
& \quad+\frac{1}{2} \mathbf{r}^{T}(\dot{\mathbf{M}}(\mathbf{q}, \Delta)-2 \mathbf{C}(\mathbf{q}, \dot{\mathbf{q}}, \Delta)) \mathbf{r} \\
& \stackrel{(2)}{=} \mathbf{r}^{T} v+\mathbf{r}^{T} \mathbf{w}\left(\mathbf{q}, \dot{\mathbf{q}}, \dot{\mathbf{q}}_{a}, \ddot{\mathbf{q}}_{a}, \mathbf{d}, \Delta\right) .
\end{aligned}
$$

Considering (12) and substituting (10), we obtain

$$
\begin{aligned}
& \dot{V}(\mathbf{r})=-\varphi(t)\|\mathbf{r}\|^{2}-\kappa(t)\|\rho([\Phi])\|\|\mathbf{r}\|^{2} \\
& +\mathbf{r}^{T} \mathbf{w}\left(\mathbf{q}, \dot{\mathbf{q}}, \dot{\mathbf{q}}_{a}, \ddot{\mathbf{q}}_{a}, \mathbf{d}, \Delta\right) \\
& \leq-\varphi_{P}\|\mathbf{r}\|^{2} \\
& -\kappa_{P}\|\rho([\Phi])\|\|\mathbf{r}\|^{2}+\|\mathbf{r}\|\left\|\mathbf{w}\left(\mathbf{q}, \dot{\mathbf{q}}, \dot{\mathbf{q}}_{a}, \ddot{\mathbf{q}}_{a}, \mathbf{d}, \Delta\right)\right\| \\
& \stackrel{\text { Lem. }}{\leq}-\varphi_{P}\|\mathbf{r}\|^{2} \\
& \underbrace{-\kappa_{P}\|\rho([\Phi])\|\|\mathbf{r}\|^{2}+\|\mathbf{r}\|\|\rho([\Phi])\|}_{=: h_{1}(\mathbf{r})} .
\end{aligned}
$$

Now, by factoring out $\|\mathbf{r}\|$ in $h_{1}(\mathbf{r})$, it is easy to see that for $\|\mathbf{r}\| \geq \frac{1}{\kappa_{P}}$ we obtain $\dot{V}(\mathbf{r})<0$ since $h_{1}(\mathbf{r}) \leq 0$. The rest of the proof is inspired by [12, Sec. 2.4.2]. Let us consider without loss of generality that the state lies outside a ball $\mathscr{B}_{\kappa_{P}^{-1}}$ of radius $\frac{1}{\kappa_{P}}$, at $t=0$. Since $\dot{V}(\mathbf{r})<0$, the trajectories will converge to the ball and there will be a finite time $t_{1}$ such that $\left\|\mathbf{r}\left(t_{1}\right)\right\|=\frac{1}{\kappa_{P}}$. Until the trajectories reach the edge of the ball, $h_{1}(\mathbf{r}) \leq 0$ and thus we can write that $\dot{V}(\mathbf{r}) \leq-\varphi_{P}\|\mathbf{r}\|^{2}$ from (13). Hence, the following holds

$$
V\left(\mathbf{r}\left(t_{1}\right)\right)-V(\mathbf{r}(0)) \leq \int_{0}^{t_{1}}-\frac{\varphi_{P}}{\kappa_{P}^{2}} d t=-t_{1} \frac{\varphi_{P}}{\kappa_{P}^{2}} .
$$

Let us now consider the following property of the inertia matrix:

$$
\lambda_{m}\|\mathbf{x}\|^{2} \leq \mathbf{x}^{T} \mathbf{M}(\mathbf{q}, \Delta) \mathbf{x} \leq \lambda_{M}\|\mathbf{x}\|^{2}, \forall \mathbf{x} \in \mathbb{R}^{N},
$$

where $\lambda_{m}=\lambda_{\min }(\mathbf{M}(\mathbf{q}, \Delta))>0$ and $\lambda_{M}=\lambda_{\max }(\mathbf{M}(\mathbf{q}, \Delta))<\infty$ represent the minimum and maximum eigenvalue of the matrix $\mathbf{M}(\mathbf{q}, \Delta)$, respectively. Recalling (11), we can write that

$$
\forall t: \quad \gamma_{1}(\mathbf{r}) \leq V(\mathbf{r}) \leq \gamma_{2}(\mathbf{r}),
$$

where $\gamma_{1}(\mathbf{r})=\frac{1}{2} \lambda_{m}\|\mathbf{r}\|^{2}$ and $\gamma_{2}(\mathbf{r})=\frac{1}{2} \lambda_{M}\|\mathbf{r}\|^{2}$. Since $V(\mathbf{r}(0)) \leq \gamma_{2}(\mathbf{r}(0))$ and $V\left(\mathbf{r}\left(t_{1}\right)\right) \geq \gamma_{1}\left(\mathbf{r}\left(t_{1}\right)\right)$, using (14) we 
have that

$$
\gamma_{1}\left(\kappa_{P}^{-1}\right) \leq V\left(\mathbf{r}\left(t_{1}\right)\right) \leq \gamma_{2}(\mathbf{r}(0))-t_{1} \frac{\varphi_{P}}{\kappa_{P}^{2}},
$$

and we can now show that $t_{1}$ is finite given that $\kappa_{P}$ is finite as

$$
t_{1} \leq \frac{1}{2} \frac{\lambda_{M} \kappa_{P}^{2}\|\mathbf{r}(0)\|^{2}-\lambda_{m}}{\varphi_{P}} .
$$

Once the trajectories entered the ball $\mathscr{B}_{\kappa_{P}^{-1}}$ they could leave it at a finite time $t_{2}$ since we have no guarantee that $\dot{V}(\mathbf{r})<0$. Let us assume that they do leave the ball, for $t>t_{2}$ the same reasoning we presented for $0 \leq t \leq t_{1}$ applies and we can therefore claim that there will be a finite $t_{3}$ at which trajectories re-enter $\mathscr{B}_{\kappa_{P}^{-1}}$. Considering (15) and the time interval for $t_{2}<t \leq t_{3}$ we have that

$$
\frac{1}{2} \lambda_{m}\|\mathbf{r}\|^{2} \leq V(\mathbf{r}(t))<V\left(\mathbf{r}\left(t_{2}\right)\right) \leq \frac{1}{2} \frac{\lambda_{M}}{\kappa_{P}^{2}},
$$

which let us conclude that the trajectories are ultimately bounded by

$$
\|\mathbf{r}\| \leq \frac{1}{\kappa_{P}} \sqrt{\frac{\lambda_{M}}{\lambda_{m}}}
$$

Corollary 1: Given that the trajectories $\mathbf{r}$ are uniformly ultimately bounded, the overall controller ultimately reaches any desired tracking performance with selection of large enough gains of the matrix $\mathbf{K}_{r}$.

Proof: This can be immediately seen observing (6) and considering $\mathbf{r}$ as the bounded input. In fact, with $\mathbf{K}_{r}$ being diagonal and positive definite, this equation represents a set of first-order linear systems that asymptotically reach $\left|\tilde{q}_{i}\right| \leq \frac{\left|r_{i}\right|}{K_{r, i}}$ for each axis $i$ [12, Proof of Th. 2.3].

Remark 1: Contrary to the robust controllers based on [15], such as [3], the tracking performance can be increased without approaching a discontinuous feedback control law using our method by increasing $\varphi_{P}, \kappa_{P}$ and/or the gains of $\mathbf{K}_{r}$. In principle, by increasing the gains the tracking error can be made arbitrarily close to zero. However, in practice there will be an upper limit of these gains due to the finite sampling rate of real applications. The maximum allowed sampling rate as well as actuator limitations have therefore a direct consequence on the maximum allowed tracking precision.

Since the trajectories of the dynamical system of (5) are uniformly ultimately bounded under the continuous control law of (10), we can now formulate the following theorem that allows us to guarantee ultimate robust performance without the need for finding specific large enough gains of $\mathbf{K}_{r}$ and the knowledge of $\lambda_{M}, \lambda_{m}$. Additionally, we also relax the assumption that the bound on the norm of the external disturbance $\left(\beta_{d}\right)$ is known.

Theorem 2: The system (1) with the continuous feedback control law composed of (4), (10) and

$$
\begin{aligned}
& \varphi(t)=\left(\varphi_{P}+\varphi_{I} \int_{0}^{t} f(\|\tilde{\mathbf{q}}\|) d t\right), \\
& \kappa(t)=\left(\kappa_{P}+\kappa_{I} \int_{0}^{t} f(\|\tilde{\mathbf{q}}\|) d t\right),
\end{aligned}
$$

where $\kappa_{I}, \varphi_{I}>0$, and

$$
f(\|\tilde{\mathbf{q}}\|)= \begin{cases}0 & \text { if }\|\tilde{\mathbf{q}}\|<\varepsilon, \\ \|\tilde{\mathbf{q}}\| & \text { otherwise }\end{cases}
$$

has ultimate robust performance for any user-defined tracking precision $\varepsilon>0$ and gain matrix $\mathbf{K}_{r}$ being diagonal and positive definite.

Proof: (sketch of proof) Assuming that the bound of the external disturbance is not known, we cannot include it in $\rho([\Phi])$. In this case, for computing $\rho([\Phi])$ based on (8) we use $[\Phi]=\mathbf{w}\left(\mathbf{q}, \dot{\mathbf{q}}, \dot{\mathbf{q}}_{a}, \ddot{\mathbf{q}}_{a}, \mathbf{0},[\Delta]\right)$. Therefore, the proof of the previous theorem for ultimate boundedness of the trajectories $\mathbf{r}$ does not directly hold. Considering (12) and noticing that $\mathbf{w}\left(\mathbf{q}, \dot{\mathbf{q}}, \dot{\mathbf{q}}_{a}, \ddot{\mathbf{q}}_{a}, \mathbf{d}, \Delta\right)=\mathbf{w}\left(\mathbf{q}, \dot{\mathbf{q}}, \dot{\mathbf{q}}_{a}, \ddot{\mathbf{q}}_{a}, \mathbf{0}, \Delta\right)-\mathbf{d}$, the derivative of the Lyapunov candidate can be written in a slightly different form:

$$
\begin{aligned}
& \dot{V}(\mathbf{r})=-\varphi(t)\|\mathbf{r}\|^{2}-\kappa(t)\|\rho([\Phi])\|\|\mathbf{r}\|^{2} \\
& +\mathbf{r}^{T} \mathbf{w}\left(\mathbf{q}, \dot{\mathbf{q}}, \dot{\mathbf{q}}_{a}, \ddot{\mathbf{q}}_{a}, \mathbf{0}, \Delta\right)-\mathbf{r}^{T} \mathbf{d} \\
& \leq-\varphi(t)\|\mathbf{r}\|^{2}-\kappa(t)\|\rho([\Phi])\|\|\mathbf{r}\|^{2} \\
& +\|\mathbf{r}\|\left\|\mathbf{w}\left(\mathbf{q}, \dot{\mathbf{q}}, \dot{\mathbf{q}}_{a}, \ddot{\mathbf{q}}_{a}, \mathbf{0}, \Delta\right)\right\|+\|\mathbf{r}\| \beta_{d} \\
& \stackrel{\text { Lem. } 1}{\leq}-\varphi(t)(1-\xi)\|\mathbf{r}\|^{2} \\
& \underbrace{-(\varphi(t) \xi+\kappa(t)\|\rho([\Phi])\|)\|\mathbf{r}\|^{2}+\left(\|\rho([\Phi])\|+\beta_{d}\right)\|\mathbf{r}\|}_{=: h_{2}(\mathbf{r})},
\end{aligned}
$$

where $\xi$ (introduced for simplifying the proof) is any scalar such that $0<\xi<1$. Now, by factoring out $\|\mathbf{r}\|$ in $h_{2}(\mathbf{r})$, it is easy to see that $h_{2}(\mathbf{r}) \leq 0$ for

$$
\|\mathbf{r}\| \geq \frac{\|\rho([\Phi])\|+\beta_{d}}{\varphi(t) \xi+\kappa(t)\|\rho([\Phi])\|} .
$$

Considering the right-hand side of (17), we have that

$$
\begin{aligned}
\forall t: \frac{\|\rho([\Phi])\|+\beta_{d}}{\varphi(t) \xi+\kappa(t)\|\rho([\Phi])\|} & \leq \max \left(\frac{\beta_{d}}{\varphi(t) \xi}, \frac{1}{\kappa(t)}\right) \\
& \leq \max \left(\frac{\beta_{d}}{\varphi_{P} \xi}, \frac{1}{\kappa_{P}}\right) .
\end{aligned}
$$

Assuming that $\beta_{d}$ is finite, it is now possible to claim that ultimate uniform boundedness of the trajectories of (5) follows as in the proof of the previous theorem once the necessary changes have been made. We proceed by contradiction. Let us suppose that

$$
\nexists t_{1}, \forall t, \quad t>t_{1}:\|\tilde{\mathbf{q}}\|<\varepsilon .
$$

Because of this assumption, the increasing functions $\kappa(t)$ and $\varphi(t)$ grow for all $t$ and consequently the maximum value of $\|\mathbf{r}\|$, such that $\dot{V}(\mathbf{r})$ is negative, decreases because $h_{2}(\mathbf{r}) \leq 0$ for $\|\mathbf{r}\| \geq \max \left(\frac{\beta_{d}}{\varphi(t) \xi}, \frac{1}{\kappa(t)}\right)$. Then, there will be a large enough $t_{2}>t_{1}$ such that for $t \geq t_{2}, \max \left(\frac{\beta_{d}}{\varphi(t) \xi}, \frac{1}{\kappa(t)}\right)$ is small enough to guarantee that the trajectories $\mathbf{r}$ ultimately stay within a ball small enough such that $\|\tilde{\mathbf{q}}\|<\varepsilon$ for any selection of the gains $\mathbf{K}_{r}$ (recalling the idea of Cor. 1), which contradicts assumption (18). 
TABLE I

DYNAMICAL PARAMETERS OF THE LINKS.

\begin{tabular}{cccl}
\hline & Link 1 & Link 2 & Uncertainty \\
\hline$m_{i}$, Mass $(\mathrm{kg})$ & 10 & $5+\delta_{m}$ & $0 \leq \delta_{m} \leq 5$ \\
$I_{i}$, Inertia $\left(\mathrm{kgm}^{2}\right)$ & $10 / 12$ & $5 / 12+\delta_{I}$ & $0 \leq \delta_{I} \leq 15 / 12$ \\
$l c_{i}$, Center of mass $(m)$ & 0.5 & $0.5+\delta_{c}$ & $0 \leq \delta_{c} \leq 0.025$
\end{tabular}

TABLE II

SIMULATION SCENARIOS

\begin{tabular}{lccc}
\hline & $\begin{array}{c}\text { Input } \\
\text { disturbance } \\
(\mathrm{Nm})\end{array}$ & $\begin{array}{c}\text { Sampling } \\
\text { time } \\
(\mathrm{s})\end{array}$ & $\begin{array}{c}\text { Required } \\
\text { performance } \\
(\mathrm{rad})\end{array}$ \\
\hline Scenario 1 (ideal) & none & none & $\|\tilde{\mathbf{q}}\|<10^{-3}$ \\
Scenario 2 & $\|\mathbf{d}\| \leq 10$ & $10^{-4}$ & $\|\tilde{\mathbf{q}}\|<10^{-2}$ \\
Scenario 3 & $\|\mathbf{d}\| \leq 10$ & $10^{-3}$ & $\|\tilde{\mathbf{q}}\|<10^{-2}$ \\
\hline
\end{tabular}

Remark 2: The implementation of our proposed controller can be easily automated since there is no need for a procedure for estimating the uncertainty bounds, e.g. the measure of the worst-case disturbance is computed automatically using interval arithmetic. Additionally, thanks to the second theorem, accurate tuning of $\mathbf{K}_{r}$ is not required since the increase of $\kappa(t)$ and $\varphi(t)$ automatically meets the tracking performance. Although this is useful by itself, it additionally opens up the possibility for on-the-fly controller design of modular and reconfigurable robots, which is possible by extending [17].

\section{SIMULATION RESULTS}

In this section, we present and discuss simulation results of the proposed control approach and a comparison with the method of Zenieh and Corless [5] that is also suitable for ultimate robust performance control. The simulations have been performed using MATLAB and Simulink R2015a and the Interval Laboratory [18] for interval arithmetic.

\section{A. RR Planar Manipulator}

We consider an exemplary two-link planar robot manipulator with revolute joints that moves in the vertical plane and thus is subject to gravity. Such a system is a suitable case study for illustrating control laws and its dynamical model is shown in fundamental textbooks such as [10, Sec. 7.4], [11, Sec. 7.3]. The graphical representation of this system as well as the components of the dynamical model with a proper factorization of the matrix $\mathbf{C}(\mathbf{q}, \dot{\mathbf{q}}, \Delta)$ can be found in [10, Sect. 7.4], which we do not replicate here for space reasons. We assume perfect knowledge of the dynamical parameters of the first link while uncertainty is assumed for the second one. The nominal values and the amount of the uncertainty is assumed as in [5]. The dynamical parameters of the links are collected in Tab. I. For completeness, we also perform simulations including sampling effects using zero-order hold to simulate more realistic scenarios. Uniformly distributed input disturbance is considered whose bound is unknown to demonstrate our method. The presented simulations include one ideal case (scenario 1) where no sampling effects and no input disturbances are considered and two additional

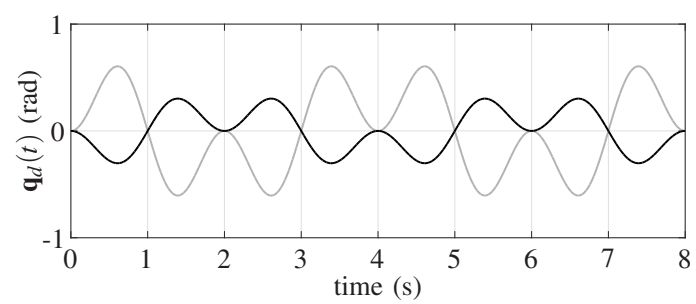

Fig. 1. Test trajectory. The first component of $\mathbf{q}_{d}(t)$ is shown in light-gray color and the second component in black.

scenarios (see Tab. II), where we consider input disturbance and sampling rates of $10 \mathrm{kHz}$ and $1 \mathrm{kHz}$ (scenario 2 and scenario 3, respectively). For simulating all scenarios, we consider that the real (unknown for control design purposes) dynamical parameters of the second link are: $m_{2}=10 \mathrm{~kg}$, $I_{2}=20 / 12 \mathrm{~kg} \mathrm{~m}^{2}$ and $l c_{2}=0.525 \mathrm{~m}$. The simulations are performed using the following test trajectory:

$\mathbf{q}_{d}(t)=\left(\frac{\pi}{4} \sin (\pi t / 2) \sin (\pi t)-\frac{\pi}{8} \sin (\pi t / 2) \sin (\pi t)\right)^{T}$,

where $\mathbf{q}_{d}(0)=\mathbf{0}$ and $\dot{\mathbf{q}}_{d}(0)=\mathbf{0}$ (see Fig. 1). Simulation results of our proposed control approach are first presented, successively we compare its performance with the $r$ - $\alpha$ tracking controller [5].

\section{B. Simulations With Our Proposed Robust Controller}

We show the behaviour of our proposed controller in Fig. 2 and Fig. 3, considering the first scenario of Tab. II for the following initial conditions:

$I C_{1}:=\left\{\begin{array}{l}\mathbf{q}(0)=\left(\begin{array}{ll}0 & 0\end{array}\right)^{T} \\ \dot{\mathbf{q}}(0)=\left(\begin{array}{ll}0 & 0\end{array}\right)^{T},\end{array} \quad I C_{2}:=\left\{\begin{array}{l}\mathbf{q}(0)=2 \varepsilon(1-1)^{T} \\ \dot{\mathbf{q}}(0)=\left(\begin{array}{ll}0 & 0\end{array}\right)^{T} .\end{array}\right.\right.$

The gains of the controller for all the simulations shown in this work have been selected as:

$$
\kappa_{P}=2, \kappa_{I}=1, \mathbf{K}_{r}=\frac{3}{\sqrt{\varepsilon}} \mathbf{I}, \varphi_{P}=1, \varphi_{I}=10^{6} .
$$

The simulation results of Fig. 2 and Fig. 3 show that the ultimate robust performance requirement considering zero and non-zero initial conditions are met. Continuity of the control law can be clearly seen by observing the evolution in time of the torque command, that in both cases is chatterfree. The simulation results with the other two scenarios are presented with the comparison in Sec.IV-D.

\section{The $r$ - $\alpha$ Tracking Controller}

We recall the essence of the control approach proposed by Zenieh and Corless in [5]. In this case, the resulting controller is remarkably simple. After a close study of the uncertain model components for obtaining proper uncertainty bounds, the control law can be implemented without online computation of the model terms. The proposed controller guarantees tracking of a desired trajectory with a prescribed rate of convergence $\alpha$ within a tolerance $r$. Since the tolerance $r$ can be selected by the user, the approach is suitable for ultimate robust performance control. Applying the approach to the considered case, the controller design 

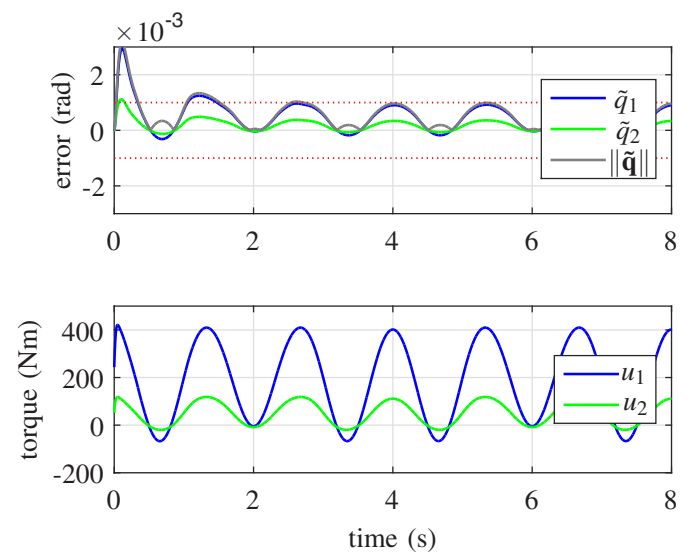

Fig. 2. Simulation with our proposed controller for scenario 1 and $I C_{1}$.

process starts from the computation of the uncertainty bounds $\beta_{i}$ for $i=0, \ldots, 3$, for all $\mathbf{q}, \dot{\mathbf{q}}, \mathbf{d} \in[\mathbf{d}]$ and $\Delta \in[\Delta]$ such that:

$$
\begin{aligned}
& \lambda_{\min }(\mathbf{M}(\mathbf{q}, \Delta)) \geq \beta_{0} \geq 0, \quad \lambda_{\max }(\mathbf{M}(\mathbf{q}, \Delta)) \leq \beta_{1}, \\
& \|\mathbf{C}(\mathbf{q}, \dot{\mathbf{q}}, \Delta)\| \leq \beta_{2}\|\dot{\mathbf{q}}\|, \quad\|\mathbf{g}(\mathbf{q}, \Delta)-\mathbf{d}\| \leq \beta_{3}(\mathbf{q}) .
\end{aligned}
$$

For the estimation of $\beta_{2}$, as proposed in [5], we consider that $\beta_{2} \geq \sqrt{\sum_{i=1}^{N}\left\|\mathbf{L}_{i}(\mathbf{q}, \Delta)\right\|^{2}}$, where $\mathbf{L}_{i}(\mathbf{q}, \Delta)$ is a square matrix such that for $\mathbf{y}:=\mathbf{C}(\mathbf{q}, \dot{\mathbf{q}}, \Delta) \mathbf{v}, y_{i}=\dot{\mathbf{q}}^{T} \mathbf{L}_{i}(\mathbf{q}, \Delta) \mathbf{v}$. To obtain an estimation of the bounds we implement a sampling procedure for $0 \leq q_{i} \leq 2 \pi,-\beta_{d} \leq d_{i} \leq \beta_{d}$ for $i=1,2,0 \leq \delta_{m} \leq 5$, $0 \leq \delta_{I} \leq 15 / 12$ and $0 \leq \delta_{c} \leq 0.025$ using $10^{5}$ samples. The procedure gives the following bounds:

$$
\beta_{0}=0.86, \beta_{1}=34.27, \beta_{2}=9.95, \beta_{3}=210.65 .
$$

Since these bounds are not formally determined, the properties of the $\mathrm{r}-\alpha$ tracking controller are not formally guaranteed. A method guaranteeing these values is not reported in the literature to the best knowledge of the authors. Once the bounds are computed, an additional tuning parameter $(\varepsilon$, that we replace by $\eta$ because it interferes with our notation), and two symmetric positive definite matrices $(\Lambda, \mathbf{Q})$ are selected such that

$$
\lambda_{\text {min }}(\Lambda) \geq \alpha, \quad \lambda_{\text {min }}(\mathbf{Q}) \geq \alpha \beta_{1}, \eta \leq(\alpha r)^{2} \lambda_{\text {min }}(\mathbf{Q}) \beta_{0} / \beta_{1} .
$$

The $r$ - $\alpha$ tracking controller can then be easily implemented as in [5]. For the simulations shown in this work the following parameters have been used:

$$
r=10^{-2}, \alpha=1, \Lambda=\mathbf{I}, \mathbf{Q}=35 \mathbf{I}, \eta=7 \cdot 10^{-5} .
$$

\section{Comparison and Discussion}

We test the two controllers using simulations for the more realistic scenarios (scenario 2 and 3) compared to the first one. The results are shown in Fig. 4 and Fig. 5. As it can be inferred from Fig. 4, the r- $\alpha$ tracking controller is conservative for the specified tracking performance behaving as a high-gain controller and letting the tracking error become far smaller than required. As a consequence, as soon as sampling effects are considered, it leads to a control command that presents the typical problem of discontinuous controllers
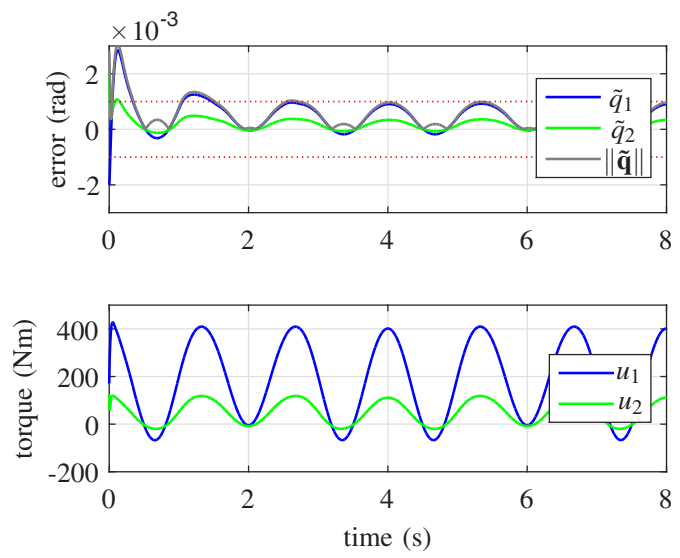

Fig. 3. Simulation with our proposed controller for scenario 1 and $I C_{2}$.

(input chattering). It should be noted that an experimental evaluation of the $\mathrm{r}-\alpha$ tracking controller was performed with a direct drive RR planar robot in [16] where this effect is not noticed. However, the system considered in this work has more uncertainty and the resulting uncertainty bounds are thus larger. Additionally, gravity in [16] is not considered since the manipulator moves in the horizontal plane. This also has a negative impact on the conservativeness of the $\mathrm{r}-\alpha$ controller in our scenarios. A system equivalent to the one that we consider is assumed in the work where the $\mathrm{r}$ $\alpha$ tracking controller is proposed [5]. However, simulation results only for ideal conditions are presented in that paper.

In contrast to the $r-\alpha$ tracking controller our proposed control law shows less conservatism, since the performance remains ultimately closer to the actual requirement. Most significantly, while the performance of the $\mathrm{r}-\alpha$ tracking controller is greatly deteriorated in scenario 2 and 3, our controller has a remarkable insensitivity to the decrease of the sampling rate while still meeting the ultimate robust performance requirement.

Our proposed method solves the discussed limitations of existing robust controllers at the price of an increased computational complexity for the on-line computation of the measure of the worst-case disturbance, using interval arithmetic. To provide a rough estimation of the computational complexity, we estimate the average time required for computing the measure of the worst-case disturbance in these simulations to be $\mu \approx 2.5 \mathrm{~ms}$ with standard deviation $\sigma \approx 0.215 \mathrm{~ms}$. The computations are run using MATLAB R2015a on a $2.7 \mathrm{GHz}$ Intel Core i7 processor with 16GB 1600 MHz DDR3 RAM running Windows 7, 64bit. It is important to notice that no particular attention has been paid to the reduction of the computational complexity for operations with interval arithmetic (e.g. parallelization and implementation in $\mathrm{C} / \mathrm{C}++$ code) since this was not the purpose of this paper and standard functions of the Interval Laboratory [18] in MATLAB scripts have been used. However, when technically realized, operations with interval arithmetic do not require considerable computational overhead with respect to standard operations as shown in Tab. 5 of [19]. 

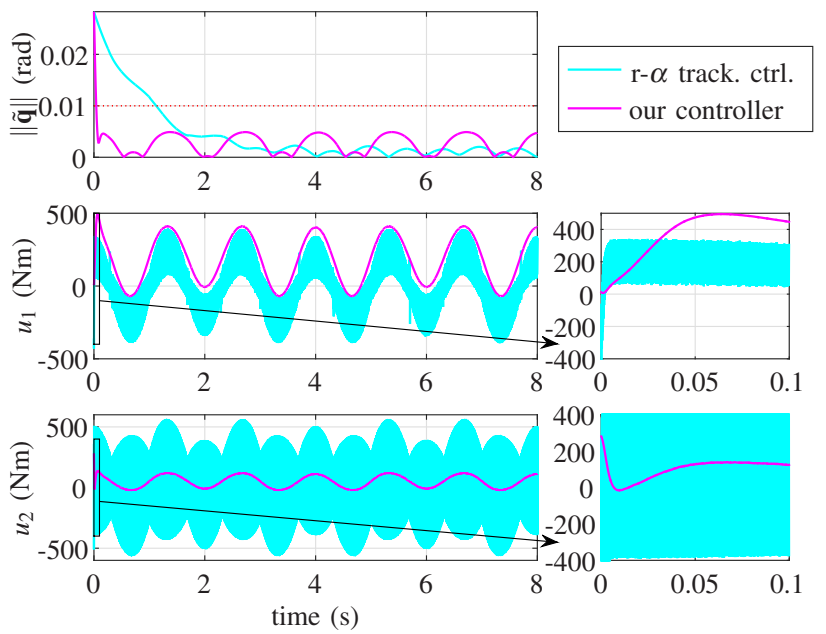

Fig. 4. Comparison of our proposed control law with the $\mathrm{r}-\alpha$ tracking controller for scenario 2 and $I C_{2}$.

\section{CONCLUSION}

An effective method for robust performance control of rigid robot manipulators is presented that allows one to overcome impractical aspects of previous methods: the disturbance bound estimation is not required thanks to the use of interval arithmetic, and the input chattering is avoided since our controller does not directly approach a discontinuous control law when increasing the desired tracking performance. Our method also guarantees the robust performance requirement to be met when considering input disturbance with unknown bounded norm. Furthermore, the introduction of the measure of the worst-case disturbance estimation and Theorem 2 makes the derivation of the robust performance control law easy to automate and enables the application to automatic controller design methods, which are especially useful for modular and reconfigurable robot manipulators. In these approaches tuning phases should be avoided or reduced, to provide easy commissioning after re-configuration. Simulations also show that our controller is superior to the r$\alpha$ tracking controller when sampling effects are considered. The application on a real and more general robotic arm will be subject of future work.

\section{ACKNOWLEDGMENT}

The research leading to these results has received funding from the People Programme (Marie Curie Actions) of the European Union's Seventh Framework Programme FP7/20072013/ under REA grant agreement number 608022 and the European Commission project UnCoVerCPS under grant number 643921.

\section{REFERENCES}

[1] T. Brogårdh, "Robot control overview: An industrial perspective," Modeling, Identification and Control, vol. 30, no. 3, p. 167, 2009.

[2] C. Abdallah, D. Dawson, P. Dorato, and M. Jamshidi, "Survey of robust control for rigid robots," Control Systems, IEEE, vol. 11, no. 2, pp. 24-30, Feb. 1991.
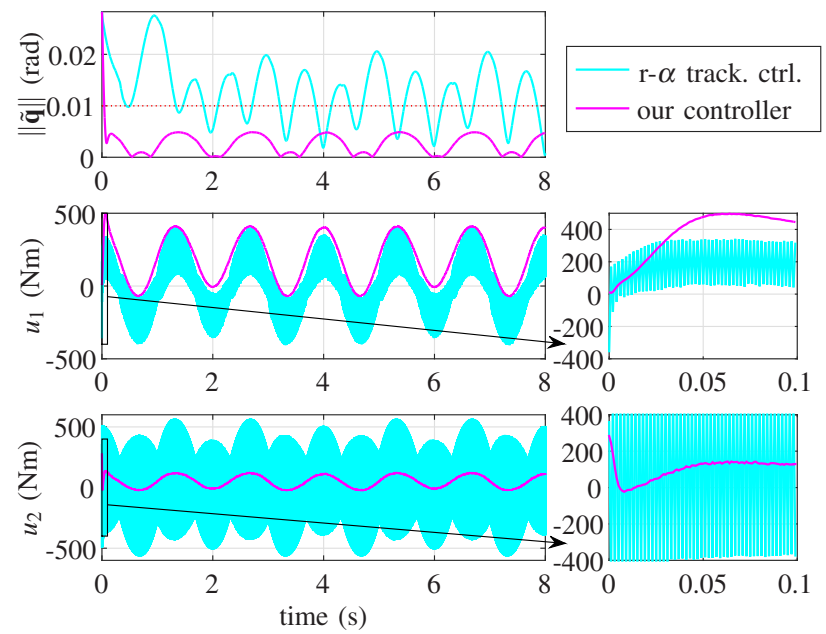

Fig. 5. Comparison of our proposed control law with the r- $\alpha$ tracking controller for scenario 3 and $I C_{2}$.

[3] M. Spong, "On the robust control of robot manipulators," IEEE Trans. on Automatic Control, vol. 37, no. 11, pp. 1782-1786, 1992.

[4] G.-J. Liu and A. Goldenberg, "On robust saturation control of robot manipulators," in Proc. of the 32nd IEEE Conference on Decision and Control, 1993, pp. 2115-2120 vol.3.

[5] S. Zenieh and M. Corless, "Simple robust $\mathrm{r}-\alpha$ tracking controllers for uncertain fully-actuated mechanical systems," ASME Journal of Dynamic Systems, Measurement, and Control, vol. 119, no. 4, pp. 821-825, 1997.

[6] F. Lin and R. Brandt, "An optimal control approach to robust control of robot manipulators," IEEE Trans. on Robotics and Automation, vol. 14, no. 1 , pp. 69-77, 1998.

[7] J. Park and W. K. Chung, "Analytic nonlinear $H_{\infty}$ inverse-optimal control for Euler-Lagrange system," IEEE Trans. on Robotics and Automation, vol. 16, no. 6, pp. 847-854, 2000.

[8] Y. Tang, M. Tomizuka, G. Guerrero, and G. Montemayor, "Decentralized robust control of mechanical systems," IEEE Trans. on Automatic Control, vol. 45, no. 4, pp. 771-776, 2000.

[9] L. Bascetta and P. Rocco, "Revising the robust-control design for rigid robot manipulators," IEEE Trans. on Robotics, vol. 26, no. 1, pp. 180187, Feb. 2010.

[10] M. W. Spong, S. Hutchinson, and M. Vidyasagar, Robot Modeling and Control. Wiley, 2006.

[11] B. Siciliano, L. Sciavicco, L. Villani, and G. Oriolo, Robotics: Modelling, Planning and Control. Springer, 2009.

[12] C. C. de Wit, B. Siciliano, and G. Bastin, Eds., Theory of Robot Control. Springer-Verlag London, 1996.

[13] W. Chung, L.-C. Fu, and S.-H. Hsu, Handbook of Robotics. Springer, 2008, book section Motion Control, pp. 133-159.

[14] S. Gutman, "Uncertain dynamical systems-A Lyapunov min-max approach," IEEE Trans. on Automatic Control, vol. 24, no. 3, pp. 437443, 1979.

[15] M. Corless and G. Leitmann, "Continuous state feedback guaranteeing uniform ultimate boundedness for uncertain dynamic systems," IEEE Trans. on Automatic Control, vol. 26, no. 5, pp. 1139-1144, 1981.

[16] A. Jaritz and M. Spong, "An experimental comparison of robust control algorithms on a direct drive manipulator," IEEE Trans. on Control Systems Technology, vol. 4, no. 6, pp. 627-640, 1996.

[17] A. Giusti and M. Althoff, "Automatic centralized controller design for modular and reconfigurable robot manipulators," in Proc. of the IEEE/RSJ Int. Conf. on Intelligent Robots and Systems, 2015, pp. 3268-3275.

[18] S. Rump, "INTLAB - INTerval LABoratory," in Developments in Reliable Computing, T. Csendes, Ed. Dordrecht: Kluwer Academic Publishers, 1999, pp. 77-104, http://www.ti3.tuhh.de/rump/.

[19] M. Schulte and E. Swartzlander, "A family of variable-precision interval arithmetic processors," IEEE Trans. on Computers, vol. 49, no. 5, pp. 387-397, 2000. 